\title{
NASA's EM Drive Thrust from the Forces of the Quantum Vacuum of Spacetime
}

\author{
Mohamed S. El Naschie \\ Distinguished Professor, Dept. of Physics, Faculty of Science, University of Alexandria, Alexandria, Egypt \\ Email: Chaossf@aol.com
}

How to cite this paper: El Naschie, M.S. (2018) NASA's EM Drive Thrust from the Forces of the Quantum Vacuum of Spacetime. Advances in Aerospace Science and Technology, 3, 1-9.

https://doi.org/10.4236/aast.2018.31001

Received: March 6, 2018

Accepted: March 12, 2018

Published: March 15, 2018

Copyright $\odot 2018$ by author and Scientific Research Publishing Inc. This work is licensed under the Creative Commons Attribution International License (CC BY 4.0).

http://creativecommons.org/licenses/by/4.0/ (c) (i) Open Access

\begin{abstract}
Building on the various manifestations of the forces latent in the quantum vacuum of spacetime such as Hawking's radiation and Unruh temperature, we resolve a major paradox connected to an immensely important proposal by NASA scientists for constructing a practically fuelless spacecraft. In a nutshell, preliminary laboratory work shows that NASA's electromagnetic drive project is viable and several experiments and measurements show it is real. Yet the proposal violates a fundamental principle of classical mechanics, namely Newton's third law. The resolution of this paradox is quite straight forward in principle. It is simply the case that although the proposal seems to be based on classical mechanics and classical thinking it is only superficially so. Deep at the roots, the EM drive proposal of NASA is not classical physics but rather based on the vacuum forces of quantum cosmology and the theory of dark energy density of the universe. In fact the proposal is deeply linked to Hawking's radiation and Unruh temperature, which is explained in some detail in the main body of the present short paper within the frame work of E-infinity Cantorian spacetime theory and D. Gross' Heterotic superstring theory. In short the quintessence of our explanation is to regard the EM drive as a quasi electromagnetic cavity with an effective event horizon akin to that of a Hawking black hole emitting radiation causing ultimately the needed thrust to push the spacecraft forwards. In addition and by invoking fractal spacetime self similarity we show that a spacecraft will be subject to another cosmic thrust on the large scale of the entire cosmos.
\end{abstract}

\section{Keywords}

EM Drive, Interstellar Flight, Vacuum Energy, Dark Energy, Cantorian Spacetime, Hartle-Hawking Wave Function of the Cosmos, Cantorian Plasma, Quantum Cosmology, Casimir-Dark Energy Nano Reactor, Heterotic Superstrings Equations of Ordinary and Dark Energy, Nonlinear Dynamics and Fractals, Hawking Radiation, Axion, Unruh Temperature 


\section{Introduction}

Newtonian mechanics as well as classical elasticity are the main analytical tools in constructing stable spacecrafts and determining its flight trajectory [1] [2] [3]. On the other hand when the travelling distance and speed are what can be described as large scale, then it becomes increasingly important to take relativistic corrections into account. By contrast quantum corrections are hardly ever needed on a realistic operational level. Contrary to the above, the present work is concerned with a situation where high energy quantum physics and cosmology as well as nonlinear dynamics and nano technology [4] play a fundamental role in the context of space flight with undreamed of possibilities as will transpire from our present analysis of NASA's EM drive project [5] [6] [7] [8].

In what follows we hope to prove that NASA's proposal for an EM drive is in principle possible [5]-[10]. To show that nothing could be simpler than appealing to the Heterotic superstring interpretation of dark energy and the Hawking radiation, Unruh temperature as well as Hartle-Hawking universe wave function theory as used in a few recent publications concerned with ordinary cosmic energy density and dark cosmic energy density [11]-[24]. The first, in the meantime well known, is the energy density of the quantum particle five dimensional zero set which amounts to $4.5 \%$ of the total while the rest of $95.5 \%$ is related to the quantum wave five dimensional empty set side of the universe [14] [15]. This gradient is sufficient to support a fuelless EM drive enabling interstellar navigation from anywhere to eternity. In fact we need an engine for navigation only, the thrust comes naturally from the local form of one sided Casimir effect of spacetime [15] [16] [17] [18]. There is a simple picture associated with the energy density gradient from $4.5 \%$ to $95.5 \%$ [8] [15] [18]. This picture is based on the quantum E-infinity fact that the quantum wave surrounds and guides the quantum particle, which is rather similar to the Bohm-Einstein pilot wave theory [11] [15]. Applying all that to the entire universe we see that a point inside the universe could be thought of as $4.5 \%$ quantum particle energy density location while any point at the hyperbolic horizon and the holographic boundary of the universe may be regarded as the $95.5 \%$ quantum wave energy density. The same idea is expressed in a different language when we invoke Dvoretzky's theorem and reason that $95.5 \%$ of the volume of the higher dimensional (i.e. 5D) universe of our real existence lies at a thin layer of the surface. Consequently we expect that any spaceship will be pulled from any point of the interior of the universe towards the hyperbolic boundary of the universe so that a skilful navigation technique could get it from anywhere to anywhere else and practically without expending much fuel except for navigation. This conclusion is reinforced completely by Dvoretzky's theorem [16] [17]. In short, NASA's proposal does not violate Newton's third law [7]. By self similarity [12]-[22] [39] the preceding picture in the large can be invoked in the small and applied to the EM drive inside the spacecraft as will be discussed later on. So much for the theoretical justification of EM drive. We stress once more that our theory goes as far as saying that 
the thrust is inbuilt into the geometry and topology of the five dimensional vacuum of a fractal spacetime so that the motion of the spacecraft is in principle possible with or without an engine. The engine is initially there mainly to steer up, down, left and right only. Thus solving the main theoretical problem is probably $1 \%$ of the difficulties. The rest of the $99 \%$ is engineering. We note on passing and in very general terms that dark energy, Hawking vacuum fluctuation, Casimir force and the like differ only with regard to boundary conditions [12]. In other words, the present theory for interstellar flight is a relative of our theory for a Casimir-dark energy nano reactor [18] [19]. For details of the exact calculation of dark matter energy and pure dark energy, the reader may consider Fig. 1 of Ref. [5] as well as the references therein. Having said all of that it is obvious to expect fro an imaginative engineering design of the EM drive to yield far more than simple steering of the spacecraft when we look upon it as something analogous to a Hawking black hole behind an event horizon so that somehow the emitted radiation could be manipulated in a way to produce the kind of thrust which does not violate Newton's third law, even in the absence of back reaction [38]. Consequently it is possible that Hawking radiation could be simulated and we could in principle produce a kind of axion dark matter-like radiation to act as an EM drive thrust which can have a net resultant force pushing the spacecraft forward. Said differently our fractal self similar universe allows for two kinds of thrust, one in the large involving the spacecraft and the entire universe and one in the small involving the EM drive inside the spacecraft only.

\section{Analysis Based on E-Infinity Theory Coupled to Heterotic Superstring Theory}

In this section we would like to show quantitatively how a back reaction in empty spacetime can never the less create a thrust, pushing a spaceship forwards without violating conservation of momentum enshrined in Newton's third law [7]. To do this we will first appeal to the fractal version of Heterotic string interpretation of the three basic cosmic energy densities, namely the ordinary (4.5\%), the pure dark (73.3\%) and the dark matter energy density (22.2\%) [16] [17] [18] [19] [20]. We recall that Einstein's maximal energy density is given via the $\mathrm{Ni}$ kolay Umov-like equation $E=k m c^{2}$ when setting $k=\gamma=1$ and finding the famous formula $E=m c^{2}$ where $E$ is the energy, $m$ is the mass and $c$ is the speed of light [21] [22]. We start here by rewriting $\gamma=1$ via the Heterotic string formalism as $\gamma=(22+k) /(22+k)$ where we have subtracted Einstein's spacetime dimensionality $D=4$ from the transfinite bosonic superstrings maximal dimensionality $26+k$ and found $26+k-4=22+k$ [16] [17] [18] [19] [20]. Here $\mathrm{k}$ is given by $\phi^{3}\left(1-\phi^{3}\right)$ which is 'tHooft's renormalon and amounts to twice the value of Hardy's probability $\phi^{5}$ where $\phi=(\sqrt{5}-1) / 2$ [16] [17] [18] [19] [20]. Proceeding in this self explanatory way by writing the three terms expression given in previous publications (see for instance Equation (8) of Ref. [21]) in a different context [18] [19] [20]. 


$$
\gamma=\frac{1+(5-\Delta)+(16+k+\Delta)}{22+k}
$$

we see that our exact ordinary cosmic energy density is given by the first term of Equation (1) [16] [17] [18] [19] [20]:

$$
\begin{aligned}
\gamma(O) & =\frac{1}{22+k} \\
& =0.04508497187 \\
& \simeq 4.5 \%
\end{aligned}
$$

The second term in Equation (1) is clearly connected to a Kaluza-Klein quantity, namely a five dimensional object or a topological mass equal 5 so the density is approximately equal $5 / 22$. However we notice an interesting coupling term $\Delta$ that will play a fundamental role in our theory and the present analysis as will shortly transpire [20] [21] [24]. The third term on the other hand is obviously connected to D. Gross et al extra bosonic dimensions of their Heterotic string theory or more accurately, the transfinite (fractal) version of the classical Heterotic theory which is found by the inclusion of the 'tHooft entangleon $k$ leading to $26+k$ and $22+k$ instead of the classical 26 and 22 [23] [24]. We see again the same $\Delta$ appearing but with a positive sign. Clearly $-\Delta+\Delta=0$ and we will dwell on the annihilation of $\Delta$ involved which is reminiscent of but by no means identical to Hawking's radiation at the edge of a black hole when interpreting $\Delta$ as an exotic virtual particle [27]. Again we recall that $\Delta$ was found to be given by $\Delta=\left(8+k^{2}\right) / 100$ and that the exact values of pure dark energy density and dark matter energy density follow from fixing this coupling term or couplion particle at this value [23] [24]. On the other hand $\Delta$ has another remarkable interpretation when $\gamma(D M)$ is rewritten in the form of first the difference between matter like densities, namely the dark matter energy density $\gamma(D M)=(22+k) / 100=0.2218033989$ and the ordinary energy density $\gamma(O)=1 /(22+k)$ as on the first line of Equation (3) and second as the difference between 5 and $(22+k)$ multiplied with the first difference as shown on the third and fourth line of the same Equation (3) [20] [21] [22] [23] [24]. Continuing in this admittedly not very obvious way to start with, one finds the exact energy density of dark matter and the meaning becomes more clear a posteriori, namely

$$
\begin{aligned}
\gamma(D M) & =\frac{[0.2218033989-(1 / 22+k)](22+k)}{22+k} \\
& =\frac{(0.17618427)(22+k)}{22+k} \\
& =\frac{3.919674776}{22+k} \\
& =\frac{5-0.08032522476}{22+k} \\
& =\frac{5-\Delta}{22+k}
\end{aligned}
$$


exactly as it should be [23] [24]. Now the same thing can be done for the pure dark energy density side involving the Gross $16+k$ bosons, namely [20] [21] [22] [23] [24]

$$
\gamma(P D)=\frac{16+k+\Delta}{22+k}
$$

Applying the above to the balance of forces acting on our "fuel-less" spaceship, we see that $\pm \Delta$, interpreted as quasi quantum particles, can solve the problems of creating a vacuum thrust pushing the spaceship "forward" without violating fundamental conservation laws of physics [5] [7]. Thus our picture in the large would entail that $+\Delta$ would be sucked by the hyperbolic horizon of pure dark energy at infinity while the $+\Delta$ gives the spaceship the needed jolt to go forward. From the preceding picture in the large we can see that by self similarity [38] [39] the suggestion in the small to regard the EM drive devise as analogous to a small black hole emitting a special type of Hawking axion-dark matter radiation is not outlandish and makes a great deal of sense [30]-[37]. We know for instance of an instructive analogy between phonons and the speed of sound on one side and photons and the speed of light on the other side which means that event horizon and its radiation are indeed not unique to black holes and may thus be drawn as we did here to explain NASA's EM drive [38].

The preceding interpretation could also be formulated using a lower dimensionality theory, namely that of a fractal Kaluza-Klein $D=5+\phi^{3}$ [22] [28]. In this case we would have the same exact densities

$$
\begin{aligned}
& \gamma(O)=\frac{\phi^{3}}{5+\phi^{3}} \\
& \gamma(D M)=\frac{1+\Delta}{5+\phi^{3}}
\end{aligned}
$$

and

$$
\gamma(P D)=\frac{4-\Delta}{5+\phi^{3}}
$$

However the coupling $\Delta$ changes to $\Delta=k-[(8+k 2) / 100] /\left(4+\phi^{3}\right)$ and the plus and minus are exchanged. This exchange of signs is harmless because in an infinite spacetime manifold plus and minus as well as up and down or left and right are all conventions and nothing more than that. At the end all what matters is that $+\Delta-\Delta=0$ and we have the vacuum thrust, which does not violate conservation of momentum [7]. However we see that not even Newton's third law could not be blindly applied when it comes to the empty set vacuum [18]-[24] and the two types of dark energy, i.e. dark matter energy and pure dark energy [26]. In the preceding analysis we made a tacit use of the Cantorian interpretation of vacuum fluctuation as well as a generalized conception of Hawking radiation without giving any details which we do however in a condensed form in the Appendix at the end of the present paper. Two more clarification remarks are in order at this point. First the geometrical shape of the EM drive should not 
rotationally symmetric in order that there can be one direction for the resultant of all the forces exerted by the Hawking-like radiation thrust. Second the magnitude of the energy density corresponding to $\Delta$ is clearly around $\Delta / 22$ which is by far smaller than the ordinary energy density of $1 / 22$ and the dark matter energy density $5 / 22$.

\section{Conclusions}

From the preceding analysis and discussion we come to the following general conclusion: when a classical mechanics design seems to violate Newtonian principles and yet we can see clearly from the experimental set up that it works, then it is surely not a classical mechanics problem and a deeper look is required to see quantum mechanical roots to it or at a minimum to realize that some nonlinear dynamics, chaos and fractals are probably involved on a fundamental level [4] [8]-[29].

Our present conclusion and resolution is the following: The quantum topology of our universe dictates that it is made up of two parts, a) a low density interior with $4.5 \%$ energy density and $b$ ) a high energy density surface with $95.5 \%$ of the total energy. This gradient is in the large what makes fuelless interstellar journeys possible so that we can state without any doubt that in principle EM drive is science and not science fiction any more. On the other hand any ordinary EM engine can be used because it is needed for navigation only. However to enhance the local cosmic thrust it may be possible by invoking self similarity [38] in the small, to design an EM drive which does exactly that along the lines explained in the analysis of Section 2 as well as the Appendix of the present work. On the other hand, the work which is concerned mainly with the engineering aspects of the present problem is in progress and we hope to communicate it in the very near future.

\section{References}

[1] El Naschie, M.S. (1976) Displacement Field in the Nonlinear Theory of Shells. AIAA Journal, 14, 1785-1787. https://doi.org/10.2514/3.7293

[2] El Naschie, M.S. (1975) Localized Diamond Shaped Buckling Pattern of Axially Compressed Cylindrical Shell. AIAA Journal, 13, 837-838. https://doi.org/10.2514/3.60451

[3] El Naschie, M.S. and Jamjoom, T.M.M. (1976) Imperfection Sensitivity and Isoperimetric Variational Problems in Stability Theory. Journal of Spacecraft and Rockets, 3, 761-763. https://doi.org/10.2514/3.27950

[4] El Naschie, M.S. (1990) Stress, Stability and Chaos in Structural Engineering: An Energy Approach. McGraw Hill, London. (International Edition 1992)

[5] MacDonald, F. (2016) It's Official: NASA's Peer-Reviewed EM Drive Paper Has Finally Been Published. Alert. https://www.sciencealert.com/it-s-official-nasa-s-peer-reviewed-em-drive-paper-ha s-finally-been-published

[6] Puthoff, H.E. and Little, S.R. (2010) Engineering the zero-point field and polarizable vacuum for interstellar flight. Cornell University Library. 
https://arxiv.org/pdf/1012.5264.pdf..

[7] Wu, C.W. (2008) Comments on Theoretical Foundation of "EM Drive". Acta Astronomica, 1-3.

[8] El Naschie, M.S. (2015) The Cantorian Monadic Plasma behind the Zero Point Vacuum Spacetime Energy. American Journal of Nano Research \& Application, 3, 66-70.

[9] El Naschie, M. and He, J.-H. (2018) Tesla's Dream from a Modern Quantum Spacetime View Point. Nonlinear Science Letters A, 9, 36-43.

[10] El Naschie, M.S. (2011) Applications of Chaos and Fractals in Fundamental Physics and Set Theoretical Resolutions of the Two-Slit Experiment and the Wave Collapse. Nonlinear Science B, 1, 1-3.

[11] El Naschie, M.S. (2013) Determining the Missing Dark Energy Density of the Cosmos from a Light Cone Exact Relativistic Analysis. Journal of Physics, 2, 19-25.

[12] El Naschie, M.S. (2015) Hubble Scale Dark Energy Meets Nano Scale Casimir Energy and the Rational of Their T-Duality and Mirror Symmetry Equivalence. World Journal of Nano Science and Engineering, 5, 57-67. https://doi.org/10.4236/wjnse.2015.53008

[13] El Naschie, M.S. (2015) An Exact Mathematical Picture of Quantum Spacetime. Advances in Pure Mathematics, 5, 560-570. https://doi.org/10.4236/apm.2015.59052

[14] El Naschie, M.S. and Helal, A. (2013) Dark Energy Explained via the Hawking-Hartle Quantum Wave and the Topology of Cosmic Crystallography. International Journal of Astronomy and Astrophysics, 3, 318-343. https://doi.org/10.4236/ijaa.2013.33037

[15] El Naschie, M.S. (2015) If Quantum "Wave" of the Universe Then Quantum "Particle" of the Universe: A Resolution of the Dark Energy Question and the Black Hole Information Paradox. International Journal of Astronomy \& Astrophysics, 5, 243-247. https://doi.org/10.4236/ijaa.2015.54027

[16] El Naschie, M.S. (2015) Application of Dvoretzky's Theorem of Measure Concentration in Physics and Cosmology. Open Journal of Microphysics, 5, 11-15. https://doi.org/10.4236/ojm.2015.52002

[17] El Naschie, M.S. (2015) Banach Spacetime-Like Dvoretzky Volume Concentration as Cosmic Holographic Dark Energy. International Journal of High Energy Physics, 2, 13-21. https://doi.org/10.11648/j.ijhep.20150201.12

[18] El Naschie, M.S. (2015) A Casimir Dark Energy Nano Reactor Design-Phase One. Natural Science, 7, 287-298. https://doi.org/10.4236/ns.2015.76032

[19] El Naschie, M.S. (2015) A Cold Fusion-Casimir Energy Nano Reactor Proposal. World Journal of Nano Science and Engineering, 5, 49-56. https://doi.org/10.4236/wjnse.2015.52007

[20] El Naschie, M.S. (2017) A Combined Heterotic String and Kähler Manifold Elucidation of Ordinary Energy, Dark Matter, Olbers's Paradox and Pure Dark Energy Density of the Cosmos. Journal of Modern Physics, 8, 1101-1118. https://doi.org/10.4236/jmp.2017.87071

[21] El Naschie, M.S. (2017) Kähler Dark Matter, Dark Energy, Cosmic Density and Their Coupling. Journal of Modern Physics, 7, 1953-1962.

[22] El Naschie, M.S. (2017) Einstein-Kaluza Combined Spacetime as the Optimal and Simplest Framework to Compute and Understand Dark Matter, Pure Dark Energy and Measurable Ordinary Energy. Natural Science, 9, 241-244. https://doi.org/10.4236/ns.2017.98024

[23] El Naschie, M.S. (2017) From the Heterotic String Quartet to the Cosmic Dark 
Matter, Dark Energy and Ordinary Energy Symphony. American Journal of Astronomy \& Astrophysics, 5, 21-24. https://doi.org/10.11648/j.ajaa.20170502.12

[24] El Naschie, M.S. (2017) Cosmic Accelerated Expansion, Dark Matter and Dark Energy from a Heterotic Superstrings Scenario. International Journal of Innovation in Science and Mathematics, 5, 53-56.

[25] Babchin, A.J. and El Naschie, M.S. (2016) On the Real Einstein Beauty E $=\mathrm{kmc}^{2}$. World Journal of Condensed Matter Physics, 6, 1-6. https://doi.org/10.4236/wjcmp.2016.61001

[26] El Naschie, M.S. (2017) The Aether as the Source of the Cosmic Dark Energy Sector. International Journal of Innovation in Science and Mathematics, 5, 177-181.

[27] El Naschie, M.S. (2013) Experimentally Based Theoretical Arguments that Unruh's Temperature, Hawking's Vacuum Fluctuation and Rindler's Wedge Are Physically Real. American Journal of Modern Physics, 2, 357-361. https://doi.org/10.11648/j.ajmp.20130206.23

[28] El Naschie, M.S. (2016) On a Fractal Version of Witten's M-Theory. International Journal of Astronomy and Astrophysics, 6, 135-144. https://doi.org/10.4236/ijaa.2016.62011

[29] Ethan Siegel: Could Dark Matter Be Powering the ED Drive? https://www.forbes.com/sites/startswithabang/2016/11/30/could-dark-matter-be-po wering-the-emdrive/\#75725c072c47.

[30] El Naschie, M.S. (2017) The Aether as the Source of the Cosmic Dark Energy Sector. International Journal of Innovation in Science and Mathematics, 5, 177-181.

[31] El Naschie, M.S. (2017) The Physics, Mathematics and Common Sense of Cosmic Dark Energy and Spacetime Extra Dimensions. International Journal of Innovations in Science and Mathematics, 5, 201-204.

[32] El Naschie, M.S. (2017) The Aether of Spacetime Physics Is the Empty Set of Pure Mathematics. Natural Science, 9, 289-292. https://doi.org/10.4236/ns.2017.99027

[33] El Naschie, M.S. (2017) Spacetime as a New Frontier Advanced Material with Applications in Physics, Engineering, Chemistry and Cosmology. Advances in Material Physics and Chemistry, 7, 347-353. https://doi.org/10.4236/ampc.2017.79027

[34] El Naschie, M.S. (2018) Super Quantization of a Cantorian Electromagnetic Field and the Cosmic Dark Energy Density of the Universe. International Journal of Innovation in Science \& Mathematics, 6, 33-37.

[35] ElNaschie, M.S. (2018) From the Logical Foundation and the Derivation of $E=\left(m c^{2} / 22\right)+\left(m c^{2}\right)(21 / 22)=m c^{2}$ to the Fractal Nature of Reality. International Journal of Applied Science and Mathematics, 5, 1-2.

[36] El Naschie, M.S. (2017) Elements of a New Set Theory Based Quantum Mechanics with Applications in High Energy Quantum Physics and Cosmology. International Journal of High Energy Physics, 4, 65-74.

[37] Marek-Crnjac, L. (2018) Quantum Entanglement and Quantum Disentanglement in Connection to the Ordinary and Dark Energy of Cosmos. In: Reimer, A., Ed., Horizons in World Physics, Nova Science Publishers, New York, Chapter 3, 91-105.

[38] Zyga, L. (2010) Physicists May Have Observed Hawking Radiation for the First Time. Physics Organization, September 28. https://phys.org/news/2010-09-physicists-hawking.html

[39] El Naschie, M.S. (2016) Einstein Dark Energy via Similarity Equivalence, 'tHooft Dimensional Regularization and Lie Symmetry Groups. International Journal of Astronomy \& Astrophysics, 6, 56-81. https://doi.org/10.4236/ijaa.2016.61005 


\section{Appendix}

We give in this appendix in a very condensed form an account of the Cantorian interpretation of quantum vacuum fluctuation as well as the generalization of Hawking radiation [27] as linked to the idea of axion holoscope theory of dark matter energy [29]. Let us recall that the Hausdorff dimension of the core of E-infinity which models our real spacetime is given by $D=1 / \phi^{3}=4+\phi^{3}=4.23606798$. The inverse is what we call universal fluctuation $\phi^{3}$ and may be understood as the surface of the empty set [13] [14] [15]. In other words, $\phi^{3}$ is a normed probability to find a Cantorian point in the Cantorian spacetime manifold while $4+\phi^{3}$ which we call the Hausdorff dimension, may be understood as an un-normed probability [8] [27] [28]. Now such a probability could be produced by union or intersections at two different points, say $\mathrm{P}_{1}$ and $\mathrm{P}_{2}$ when it is dissected to $\phi^{2}$ multiplied with $\phi$ where $\phi^{2}$ is the empty set quantum wave and $\phi$ is the zero set quantum particle [8]. Seen that way two $\phi^{2}$ at the two different points $\mathrm{P}_{1}$ and $\mathrm{P}_{2}$ intersect and give us Unruh temperature $\phi^{2} \phi^{2}=\phi^{4}$ while the two zero sets $\phi$ and $\phi$ intersect and give us the empty set Aether [30]-[37]. This process takes place all the time and everywhere in our spacetime manifold leading to the sizzling process which we call volume fluctuation. Now similar to the edge of a black hole two different zero set particles, say $\phi^{(-)}$and $\phi^{(+)}$behave in an analogous way to Hawking's radiation scenario [27] at an electromagnetic cavity [29] representing the exhaust of NASA's ED-drive [29]. Continuing the preceding picture to its ultimate logical conclusion, we arrive at the remarkable axion dark matter experiment (ADMX) which was introduced in physics around 1983 by Pierre Sikivie to explain dark matter energy [29] long before thinking about any fuelless ED-drive spacecraft. Consequently we could regard $+\Delta$ as a Hawking particle sucked into the cavity while the $-\Delta$ is the other Hawking particle which escapes from the exhaust as radiation giving the spacecraft the thrust which was measured in various experiments mentioned in the relevant literature [5] [6] [7] [29]. In short NASA's EM drive is analogous to Hawking's black hole which, as we stressed earlier on, is not surprising because an even horizon is not unique to black holes and there are well known experiments in which something resembling Hawking radiation is produced without a real black hole using laboratory simulations [38]. In the preceding picture we considered the thrust, produces on the small scale by means of the EM drive devise. However by self similarity [38], the same picture holds on the large scale involving the entire universe. In other words our spacecraft is acted upon by two reactionless thrusts. The first is due to the large scale structure of the entire expanding universe and the second is by self similarity due to the forces produced on the much smaller scale by the EM drive. 